\title{
Enabling Personal Computational Handweaving with a Low-Cost Jacquard Loom
}

\author{
Lea Albaugh \\ Carnegie Mellon University \\ Human-Computer Interaction Institute \\ lea@cs.cmu.edu \\ Lining Yao \\ Carnegie Mellon University \\ Human-Computer Interaction Institute \\ liningy@cs.cmu.edu
}

\author{
James McCann \\ Carnegie Mellon University \\ Robotics Institute \\ jmccann@cs.cmu.edu \\ Scott Hudson \\ Carnegie Mellon University \\ Human-Computer Interaction Institute \\ scott.hudson@cs.cmu.edu
}

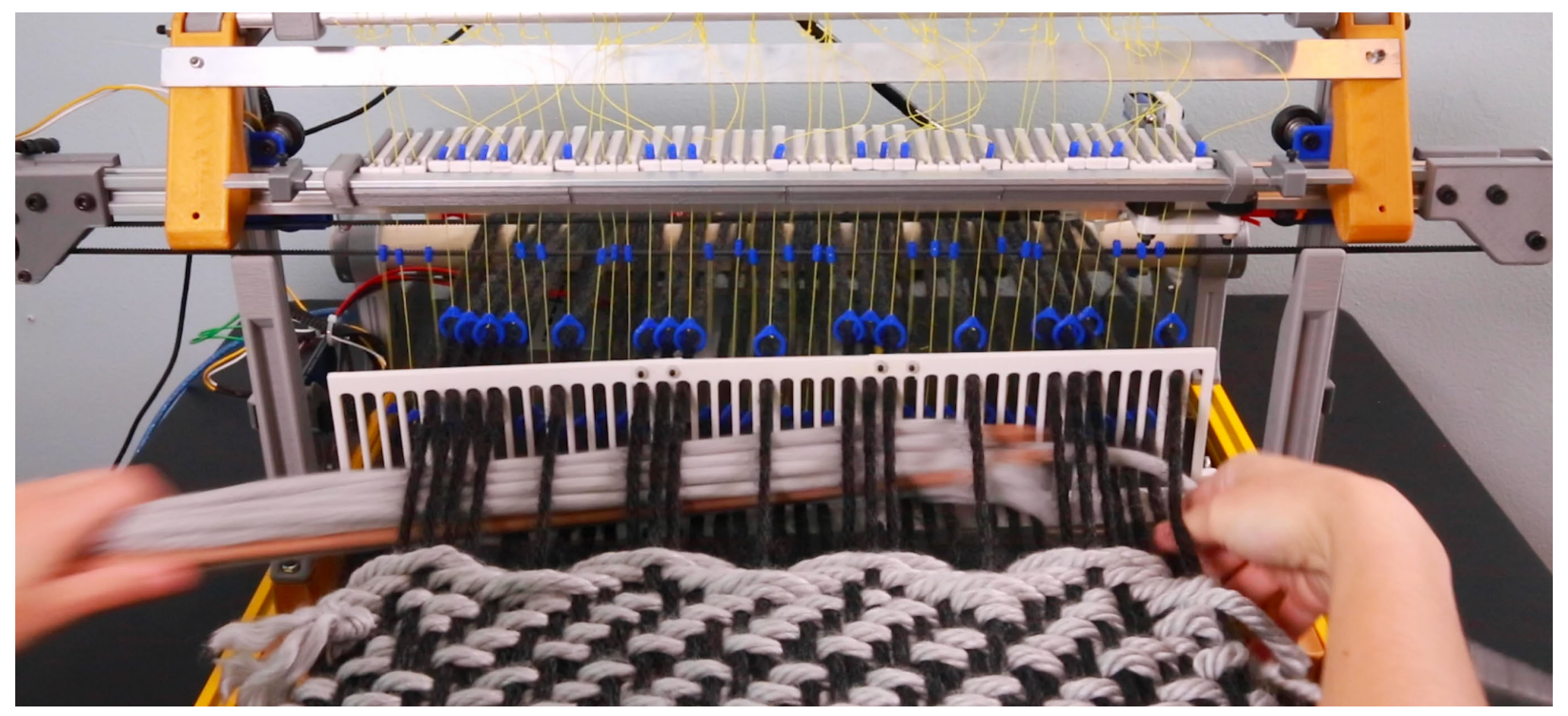

Figure 1: Tabletop jacquard weaving.

\begin{abstract}
We present an inexpensive tabletop loom that offers fully computational patterning while maintaining the flexibility of handweaving. Our loom can be assembled for under US $\$ 200$ with 3D printed parts, and it can be controlled straightforwardly over USB. Our loom is explicitly a hand loom: that is, a weaver is required to operate the weaving process and may mediate row-by-row patterning and material specifics like yarn tension. Our approach combines the flexibility of fully analog handweaving with the computational affordances of digital fabrication: it enables the incorporation of special techniques and materials, as well as allowing for the possibility
\end{abstract}

\section{(c) (1) (2)}

This work is licensed under a Creative Commons

Attribution-NonCommercial-ShareAlike International 4.0 License.

CHI '21, May 8-13, 2021, Yokohama, Japan

(c) 2021 Copyright held by the owner/author(s).

ACM ISBN 978-1-4503-8096-6/21/05.

https://doi.org/10.1145/3411764.3445750 of computational and creative interventions in the weaving process itself. In taking this approach, we aim to serve a range of end users including artisans and researchers, whether for skill-building, for rapid prototyping, or for creative reflection.

We describe the mechanical and electronic implementation of our loom and show examples of its use for personal fabrication.

\section{CCS CONCEPTS}

- Human-centered computing $\rightarrow$ Human computer interaction (HCI).

\section{KEYWORDS}

Textiles; weaving

\section{ACM Reference Format:}

Lea Albaugh, James McCann, Lining Yao, and Scott Hudson. 2021. Enabling Personal Computational Handweaving with a Low-Cost Jacquard Loom. In CHI Conference on Human Factors in Computing Systems (CHI '21), May 8-13, 2021, Yokohama, Japan. ACM, New York, NY, USA, 10 pages. https: //doi.org/10.1145/3411764.3445750 


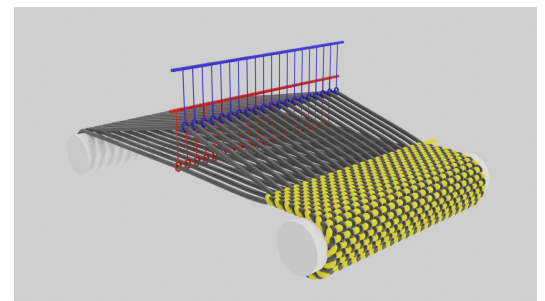

(a) Loom with two sets and "plain weave" fabric

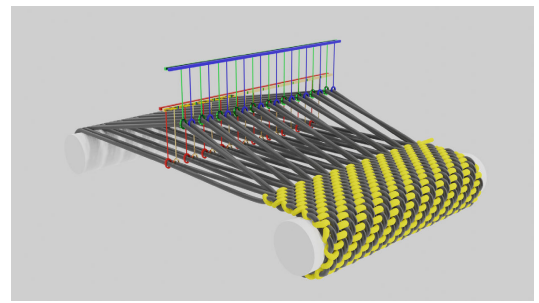

(b) Loom with four sets; two are shown raised

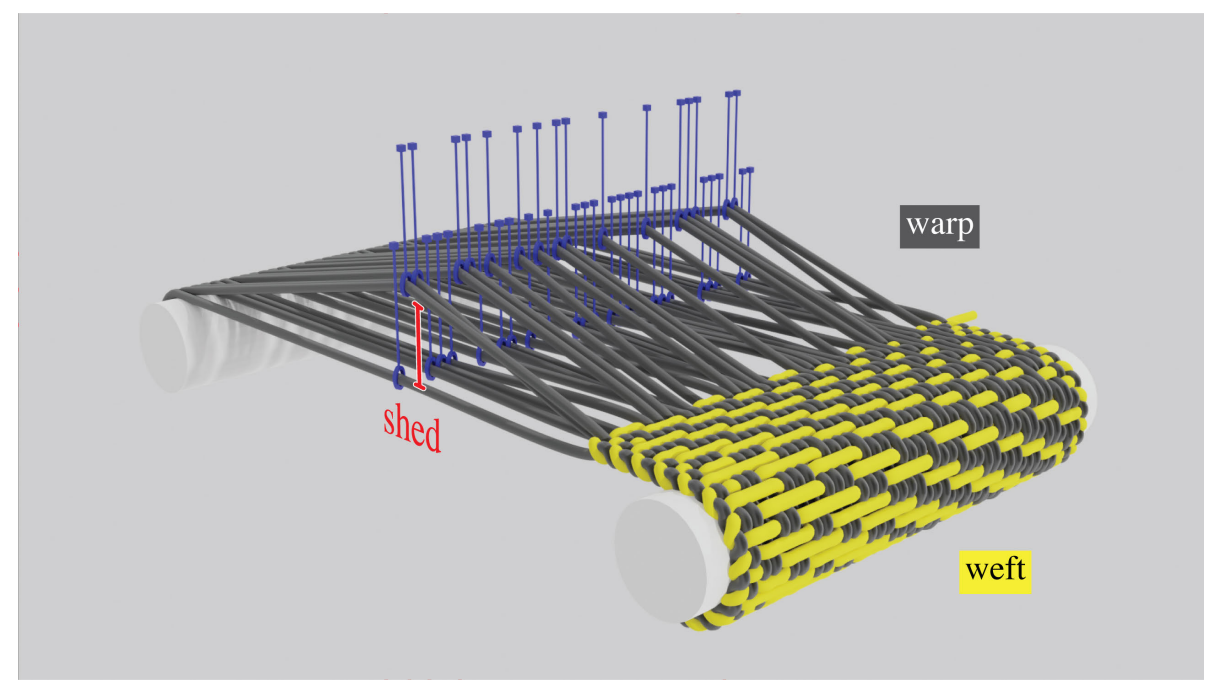

(c) "Full jacquard" loom

Figure 2: Basic loom functionality and terms.

\section{INTRODUCTION}

Weaving is one of humanity's oldest technologies, with variants across human cultures and time. Its history is intertwined with politics at the global scale and the domestic one: woven fabrics are in our clothes and our homes, as well as our agricultural geotextiles and our aerospace carbon-fiber hulls.

The pattern of the weave itself can determine many material properties of a woven fabric. The weave pattern can set the appearance, including, e.g., matte velvet, shiny satin, and vibrant multi-color patterns; change the feel of the fabric, from, e.g., rough plain weave to fuzzy terry; and it can even change the topology, with, e.g., double-cloth weaves allowing for integrated tubes and pockets. All of these various patterning techniques can be combined within a single woven item, making weaving a very flexible meta-material.

As a fabrication technology, weaving is remarkable for the range of possible tool setups, from the simplest hand-held peg or backstrap loom to multi-story industrial powerlooms. Each type of loom has its benefits and trade-offs, but typically looms which support computational patterning (flexible, reconfigurable pattern selection that doesn't rely exclusively on human memory and dexterity or a fixed set of mechanical elements) are positioned as expensive, large machines for automated industrial use. At the other end of the scale, hand looms that are suitable for prototyping, experimentation, and playful or meditative use may not support complex patterning, or may require a great deal of expertise or effort to achieve these effects. In the middle, we see "production prototyping" devices such as the TC2 Jacquard Loom [15], which strike a balance between the automation of an industrial loom and the flexibility of a hand loom. However, these looms are still too expensive and large - the TC2 has a baseline cost of approximately $\$ 30,000$ USD for their smallest model, which is 61 inches tall with a 58 inch by 48 inch footprint and additionally requires an air compressor - to be practical outside of dedicated spaces such as fashion schools and textiles-specific laboratories. Additionally, even these relatively simpler looms are still complex and difficult to maintain, and their mechanical complexity scales with the width of the fabric (counted in number of threads).

We present an inexpensive tabletop loom that offers fully computational patterning, while maintaining the flexibility of handweaving. Our loom can be assembled for under \$200 USD with 3D printed parts, and it can be controlled straightforwardly over USB. Our loom is explicitly a hand loom: that is, a weaver is required to operate the weaving process and may mediate row-by-row patterning and material specifics like yarn tension. This approach therefore combines the flexibility of fully analog handweaving with the computational affordances of digital fabrication: it enables the incorporation of special techniques and materials, as well as allowing for the possibility of computational and creative interventions in the weaving process itself - for skill-building, for interactive design, or for creative reflection. In taking this approach, we aim to serve a range of end users including artisans and researchers, whether for exploring expressive weaving or prototyping e-textile or composite material applications.

We describe the mechanical and electronic implementation of our loom and show examples of its use for personal fabrication.

\subsection{Jacquard Weaving}

Weaving is a process of interleaving threads; most looms enable this process by doing two things. First, they hold a set of threads (the warp threads) parallel and at tension. Second, they selectively raise or lower some of those warp threads, forming a gap known as the shed, allowing the weft to interlace.

The first task is reasonably straightforward. Minimal solutions include the peg frames common in tapestry handweaving as well as simply tying the warp between the weaver's body and a nearby stationary object (a "backstrap loom"), but most production looms include rolling beams that longer warps can be wound around. 
The second task-warp selection and forming a shed-has been accomplished by a great variety of mechanisms. Simpler looms, like most hobbyist looms, can only select amongst a number of sets of warp threads determined by the quantity of "frames" (or "harnesses"/"shafts" depending on the specific mechanism) available on the loom. For example, a very basic loom might support only two sets, as in Figure 2(a). A weaver might allocate all the evennumbered threads to one set, and all the odd-numbered ones to the other; at weaving time, that weaver can only alternate selection between those two sets, producing the "plain weave" structure. A more typical number of frames is four (Figure 2(b)) or eight. These looms occasionally (but rarely) have up to twenty-four frames, at the high end often requiring the support of an analog or electronic "dobby" mechanism to keep track of which sets should be selected in a row. Designing a weaving pattern for these looms is an expert task which is constrained by the loom's setup; changing the setup is labor-intensive and is typically only done when an entirely new fabric is begun.

In contrast, a "full jacquard" loom is one in which every warp thread can be selected individually. This style of loom is commonly attributed to Joseph Marie Jacquard, whose 1804 model used punched cards to control the selection of each pattern row [16]; related concepts were considered even earlier, including inventions by Basile Bouchon in 1725, Jean Baptiste Falcon in 1728, and Jacques Vaucanson in 1740 [39]. Electronic versions of the jacquard loom, with computer-controlled solenoids standing in for the punched cards, are used heavily in today's global textiles industry. Industrially, the flexibility of jacquard warp selection is valuable because a different weaving pattern can be deployed without the setup overhead that would be required to change patterns on a less-complex loom. (This setup time can be measured in days or even weeks for an industrial machine.) Our loom retains the advantage of low setup overhead per-pattern, and in the handweaving context this flexibility is even more powerful: it can extend to row-by-row design decisions within a composition, in real time alongside the weaving process.

\subsection{Related Work}

The production of textiles is an ancient form of technology which significantly predates recorded history. For example, recent physical evidence has been uncovered that points to the use of twisted fibers even by Neanderthals [21], and other direct evidence suggests that human weaving dates to at least 25,000 years ago [46]. As a result, the technology of weaving is widely dispersed across both time and cultures. Notably, the invention of a series of increasingly automated weaving machines formed the functional and symbolic heart of the early stages of the Industrial Revolution. The jacquard loom in particular holds an important place in the history of computing by including both a stored numerical representation of a task, and a mechanism for interpreting that representation to automatically carry out a sequence of actions [16, 41]. This fundamentally computational approach allowed the jacquard loom to escape from the confines of a limited set of actions (in this case a pre-determined set of shed patterns) to a more general and complete capability, dramatically expanding the range of expression to include all possible shed patterns.
Table 1: Handloom Comparison

\begin{tabular}{c|c|c}
\hline Loom & Selection Type & Size \\
\hline AVL Jac3g [8] & parallel jacquard (solenoids) & floor \\
TC2 [15] & parallel jacquard (solenoids) & floor \\
OSLoom [9] & parallel jacquard (muscle wire) & floor \\
Little Weaver [6] & $18-24$ frames & tabletop \\
Liou [28] & parallel jacquard (motors) & tabletop \\
Moyer [33] & parallel jacquard (solenoids) & tabletop \\
Schaefer [43] & serial jacquard (cams) & tabletop \\
Nicholls [35] & serial jacquard (Lego ${ }^{\mathrm{TM}}$ ) & tabletop \\
Ruta [37] & manual jacquard pins & tabletop
\end{tabular}

\subsection{Contemporary Handlooms}

Industrial looms are as fully-automatic as possible; in addition to the selection and shedding described in Section 1.1, an industrial loom inserts and compresses the weft, manages spooling and un-spooling the warp and the cloth as it is formed, and may even post-process the fabric in specialized applications (e.g. cutting apart velvet [50]).

In contrast, a handloom requires a weaver to insert and compress the weft, as well as to attend to other matters of craft such as managing tension (to a greater or lesser extent depending on the specific loom). Handweaving therefore does not require the loom to be tuned to produce a specific kind of fabric, and also offers opportunities for creative improvisation [3].

Table 1 summarizes the landscape of contemporary computational handlooms. Entries in bold are (or have been) commercially available; other entries are personal, research, or incomplete/deprecated projects. "Floor" looms are large, standalone machines typically intended to produce full-width fabric, whereas tabletop looms are small and deployable. The form factor has implications for both price and intended use.

Two full jacquard handlooms are currently commercially available: the Jacq3g loom by AVL Looms [8] and the TC2 loom by Digital Weaving Norway [15]. Both are "prosumer" floor looms intended for either production prototyping or serious artisanal practice, and both use a parallel selection mechanism similar to industrial looms. The Jacq3g uses solenoids to displace hooks onto a lifting bar; the baseline model has a 45 inch by 84 inch footprint ( 90 inches tall) and costs approximately $\$ 28,000$ USD. The TC2 uses electronic valves to direct air from a compressor; the baseline model has a 58 inch by 48 inch footprint (61 inches tall) and costs approximately \$30,000 USD. The OSLoom project was Kickstarted in 2010 with the intention to build a floor loom using parallel muscle wire selection. While a full OSLoom was never built, the approach has similar power, size, and electrical complexity drawbacks for home or casual use.

AVL's smaller "Little Weaver" approaches ours in intent, as it is a portable (tabletop) computational loom which is designed to easily integrate with a home computer setup, such as by allowing Wi-Fi connectivity [6]. However, the Little Weaver is a frame loom with eight, sixteen, or twenty-four frames, not a full jacquard loom. When they were last sold, Little Weaver looms cost \$2,000-3,000, depending on the number of frames [7].

Many personal hobby projects have approached loom design and construction. These have been greatly successful in the area of looms which are not computationally controlled, such as DIY 
pocket tapestry looms [17] and small frame looms [11, 52]. Hobby jacquard looms have been periodically explored, but these projects typically still use parallel selection strategies [28, 33]. Of particular note are two designs which use a serial actuation strategy similar to ours: a device by Schaefer which uses set of circular cams actuated by a shaft moved along the heddles [43], and the Weav3r loom constructed with Lego ${ }^{\mathrm{TM}}$ blocks[35].

Lastly, the Ruta loom by Ooms et al [37] is an interesting byway in contemporary loom design which centers hands-on sensemaking for novice weavers. It is fully jacquard in the sense that it can select any warp set, but it is entirely non-electronic: the weaver programs each set by manually arranging pins in a frame. We see the manual override capability of our loom as potentially offering similar opportunities for hands-on engagement.

\subsection{Computational Craft Practice}

In comparison to non-computational weaving, we believe that computational mediation is not simply faster, but potentially a qualitatively different experience from unmediated weaving. The line between computational and craft practices has always been blurred $[27,42]$; within weaving, contemporary practices incorporate lasercutting [1, 11, 52], glitch [30], and Photoshop-based design techniques [45]. The introduction of weave planning software and even home electronic dobby looms in the 1980's spurred new capabilities [51] and even new aesthetics of handweaving [44].

Within human-computer interaction, computational craft practice has inspired a number of projects including digital woodworking systems [29, 48, 54], augmented hand tools [53], and even fabrication-based computer games [2, 47]. In its relatively small size and low cost, we see our loom as part of the movement toward "personal fabrication" [34]; in our emphasis on flexible use scenarios, including some quite idiosyncratic ones, we draw inspiration from "digital craftsmachineship" [5, 38] and hybrid fabrication [14, 49].

On the software side of things, related work includes AdaCAD [19] and special-purpose tools for 3d weaving [22]. We take inspiration from these systems as well as broader on-machine paradigms such as the CopyCAD [18] and Sketch 'n' Stitch [20] systems which situate computational design explicitly in their output medium e.g., our "brushes" input is a departure from both expert planning applications and simplified pixel-input editors in combining correct-byconstruction pattern tooling with thread-level overrides.

\section{TECHNICAL IMPLEMENTATION}

The main unique design feature of our loom is the mechanism by which it forms its shed: the gap between upper and lower warp threads through which the weft passes to create interlacing.

Typical electronic jacquard-style looms form a shed in two stages: selecting a number of warp threads, then raising (or, depending on other factors of the loom's geometry, lowering) the selected threads. Because they are designed for industrial production weaving, these looms prioritize very high production speeds; to achieve such speeds, industrial looms must do the entire row's selection at the same time, requiring an electronic actuator (typically a solenoid) for each individual warp thread. The number of solenoids, as well as the power requirements and the complexity of the control circuitry, must therefore grow with the number of warp threads.

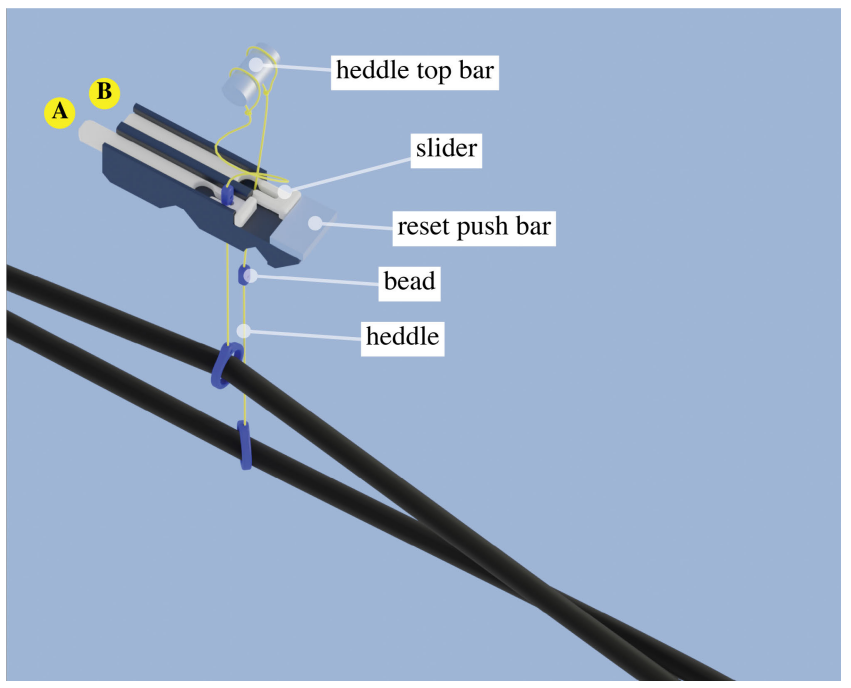

Figure 3: The heddle mechanism. Slider $A$ is in the reset position, with its heddle raised. Slider $B$ has been pushed forward, and its heddle has dropped.

In contrast, our loom differs from these by performing the electronic selection serially using a passive bistable shedding mechanism. With this design, a single solenoid mounted on a drive belt can perform selection on any number of warp threads.

This arrangement greatly lowers the initial parts cost, maintenance difficulty and costs, the complexity of electronic wiring and power requirements, and the overall size and weight of the loom. With access to a hobbyist FDM 3D printer, our loom can be made for under US\$200, and its "table loom" form factor fits on a desk or countertop.

\subsection{Heddle Design}

The heart of any warp selection mechanism is the heddle. Each heddle guides the position of a single warp thread; heddles can be selected and raised (or for some looms lowered), taking their warps along with them to form the shed. Heddles are commonly made of wire or string (or thin wood/plastic, in the case of more limited "rigid heddle" looms which can only actuate a fixed pair of shed patterns).

Our design uses an array of string heddles which are fixed at the top and hold their warp threads at the bottom, Figure 3. During the selection process, all heddles are initially raised, lifting each corresponding warp thread and providing a small amount of tension on the heddle. Each heddle has a "bead" which is 3D printed onto it, and each passes through a keyhole-shaped hole in its own “selection slider." At each slider's home position, its heddle's bead is held captive in the narrow slot part of the keyhole. When a slider is pushed forward, gravity and warp tension pull the bead down through the hole, allowing the heddle and its warp thread to drop.

When selection is complete, the warp threads corresponding to each actuated slider will have dropped down, while all remaining warps will remain pulled high, thus forming the desired shed. 


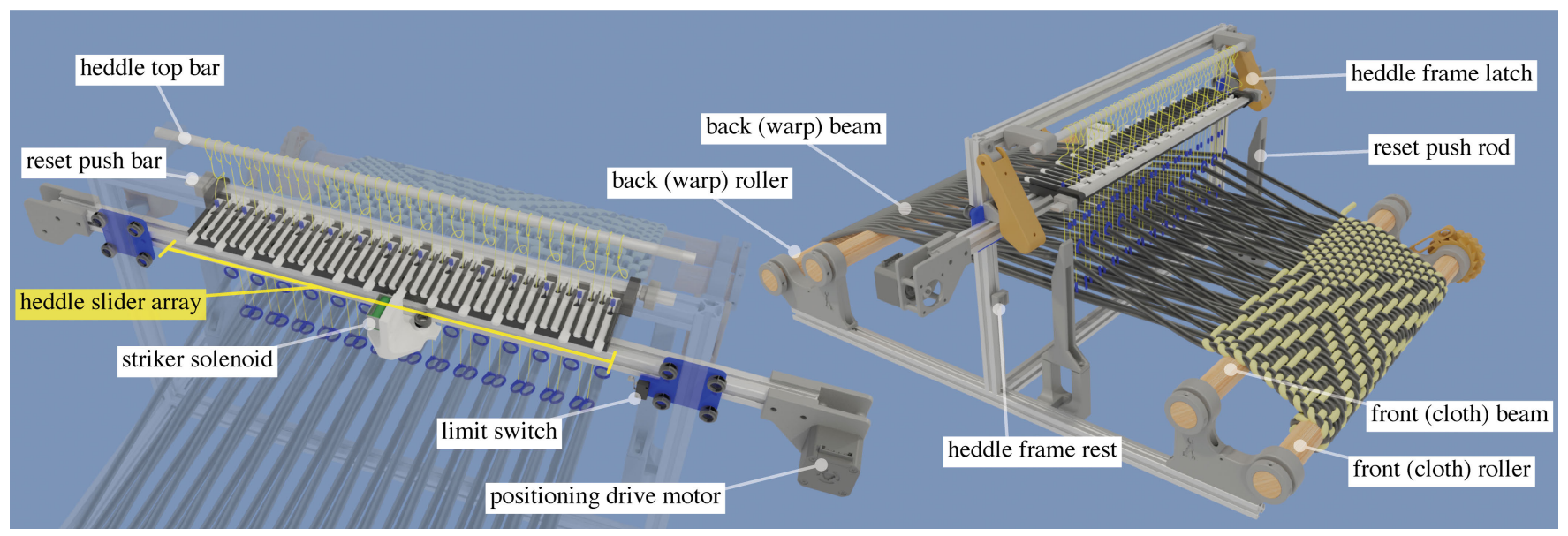

Figure 4: The main components of the loom.

\subsection{Frame and Tensioning System}

These heddle mechanism can easily be operated by hand, but our jacquard heddle frame incorporates a stepper motor and belt assembly which positions a solenoid sequentially to strike each selected heddle.

We used a motor and belt assembly common to 3D printer construction to ensure the availability of parts. Our loom is designed to be flexible about specific components; e.g. any push solenoid with approximately one centimeter of throw would be compatible, as would any NEMA 17 stepper motor. We specifically used a NEMA 17 bipolar stepper motor with $1.8^{\circ}$ step size rated at $0.59 \mathrm{Nm}$ holding torque (2.0A per phase), with a GT2 (2mm pitch) timing belt and a 12-tooth GT2 drive pulley. The belt drives a linear motion platform consisting of $24 \mathrm{~mm}$ OD Delrin v-wheels with \#625 (5 x $16 \times 5 \mathrm{~mm}$ ) bearings. The platform rides on the same $20 \mathrm{~mm} \times 20 \mathrm{~mm}$-slot aluminum extrusion that the heddle mechanism array is mounted to. Our solenoid is an XRN-0530 pull-type solenoid with $10 \mathrm{~mm}$ throw (rated at $12 \mathrm{v} 0.3 \mathrm{~A}$ ).

The frame additionally includes a reset push rod which, acting with the reset push bars, can reset the entire array of heddle sliders. The heddle frame is either held in the upper position by a pair of frame latches, or rests in the lower position on frame rest brackets.

The rest of the loom handles warp and cloth storage and tension, and it closely mirrors established home loom designs. (It may be possible to retrofit an existing loom to reduce build complexity.) Four wooden dowels comprise the rollers and beams. Warp threads are wound onto a warp roller at the back of the loom, pass over the warp beam, through the heddles, over the cloth beam and are tied onto the cloth roller. As it is woven, fabric can be progressively wound onto the cloth roller as warps are unwound from the warp roller. Ratchets on the warp and cloth rollers maintain tension on the warp.

Between the heddles and the cloth beam, warp threads pass through a reed, which the weaver uses to compress each weft against the fabric as it is woven.

\subsection{Control Electronics}

The control electronics for our loom are provided by the RepRap Arduino Mega Pololu Shield (RAMPS) platform [26], a popular and inexpensive choice for hobby projects involving dimensional motor control, such as 3D printers and pen plotters. The RAMPS platform incorporates a programmable micro-controller, power management, stepper controllers, and MOSFET switched high current drives. This board controls a single bipolar stepper motor of the type used in most inexpensive 3D printers, along with a small solenoid (a small "flyback" diode across this inductive load is also added to protect the MOSFET drive transistor from transients). Finally, an endstop switch is connected to the controller board to allow the loom to place the heddle selection head at a known "home" position. All other positions are determined in low-level firmware by counting steps of the stepper motor with respect to this position. Off-theshelf open source firmware (a specially configured, but unmodified copy of the Repetier v0.92 3D printer control firmware [23, 24]) is loaded into the RAMPS board and accepts G-Code commands over a serial connection to drive the motor and solenoid. We use this system to reliably direct the selection head to exact heddle locations, and selectively power the solenoid to push forward selected sliders.

\subsection{Input: Computer Vision}

An advantage of our heddle design is that it can be easily overridden: the weaver can manually drop or lift heddles to tinker with the pattern directly on the loom. As a computational system, this tinkering is especially valuable as input: the weaver's choices can be recorded, transmitted, or act as a basis for future patterning.

To avoid adding electrical complexity to the loom hardware, we used a webcam and computer vision techniques to sense the heddle positions. We mounted a Logitech C922 webcam to the main loom frame such that the camera lens was $50 \mathrm{~cm}$ above the warp, Figure 5(a). This position gives the camera a field of view which extends to the front of the loom. (An additional benefit of this input approach is that the weaving area can be used for other visual input as well. We use this capability in our "On-Loom Input" example described in section 3.1.) 


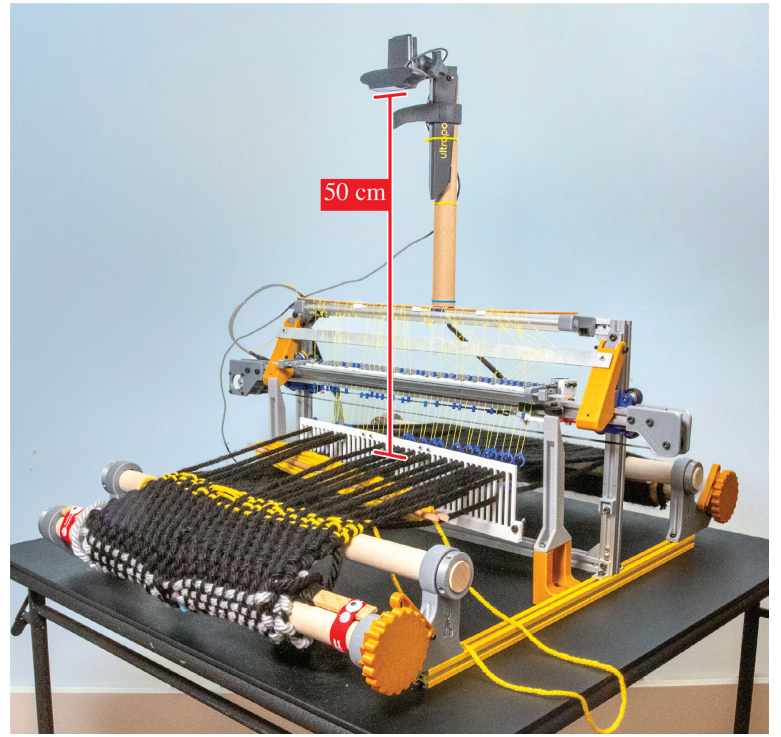

(a) The camera mount

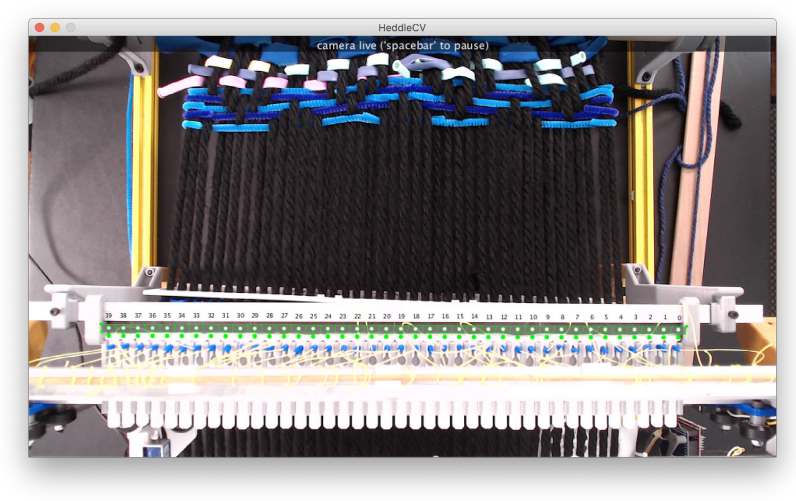

(b) The view from the camera. Green lines show the computer vision's region of interest for each heddle slider.

Figure 5: Heddle input using OpenCV.

We use OpenCV to determine the heddles' positions. To do so, our region of interest is the area just in front of the heddles' home positions, Figure 5(b), which will show the color of the bed if the slider is in its home position, or the color of the slider if it is moved forward. To calibrate the system, the weaver clicks on the four corners of this area in a live camera view. (This calibration is persistent across weaving sessions as long as the camera isn't moved.) The system divides the area into a number of perspective-corrected cells corresponding to the number of heddles, then calculates an average pixel value in each cell. These averages are then thresholded with Otsu's method. A cell that is mostly white indicates that the slider is forward, and therefore its heddle is dropped.

For maximum visual contrast, we printed the heddle sliders in white and colored the slider bed black; we additionally enable the built-in lighting of the webcam to reduce shadows. These measures, along with the adaptive thresholding, ensure that the system is robust in typical room-lighting conditions.

\subsection{Control Software and Backend}

We use Processing to interface with the loom hardware. The main control software consists of several modules: communication over a USB serial port with the RAMPS control board, webcam capture and computer vision, and networking to connect to server-based design tools (such as our "Remote Collaboration" example). For interoperability, weaving patterns are consistently passed as lists of pattern rows, with " 1 " indicating a raised warp and " 0 " indicating a dropped one. We describe some specific design interfaces in Section 3.

\subsection{Operation}

For each row of weaving, the Processing sketch fetches the next row of the weaving pattern from either local or networked design software. This pattern row is converted to G-code commands which sequentially position the selector solenoid at each heddle which should be dropped, then strike with the solenoid to hit the slider and drop the heddle. The weaver may then modify the shed as desired, and the computer vision system can record these alterations. Then, the weaver passes the weft of their choice through the open shed, and uses the reed to "beat" the fabric, pushing the latest weft into the fabric being formed.

To reset all the heddles, the weaver lowers the heddle frame. After the sliders have passed below any dropped heddle beads, the reset push-rods engage the reset bar to push all sliders back into their home positions, trapping each bead above its slider. At this stage, the weaver also periodically takes up the completed fabric onto a roller at the front of the loom and releases an equivalent length of warp threads from a similar roller at the back of the loom.

Finally, the weaver can raise the heddle frame again to prepare for another row of weaving.

\section{PERSONAL WEAVING SYSTEMS}

The low cost and small footprint of our loom makes it suitable for personal weaving practice. We describe three systems which make use of specific capabilities of tabletop jacquard handweaving. The software interactions align with the principles guiding the loom itself: flexibility and extensibility. In taking this approach, we aim to serve a range of end users including artisans and researchers, whether for exploring expressive weaving or prototyping e-textile or composite material applications.

\subsection{On-Loom Input}

An advantage of handweaving is the ability to incorporate disparate materials; jacquard handweaving in particular can use "double cloth" patterns to form two layers of fabric which can act as a pocket.

Double cloth is a pattern type in which an area of the fabric has two separate faces, Figure 6. In this area, half of the warp threads are allocated to one face, and the other half are allocated to the other; the weft only interlaces with one face per pass. Double cloth patterns are a popular way to produce areas of solid color [32], as well as, in the hands of a skilled weaver, a way of producing complex intersecting topologies [36]. However, designing a double 


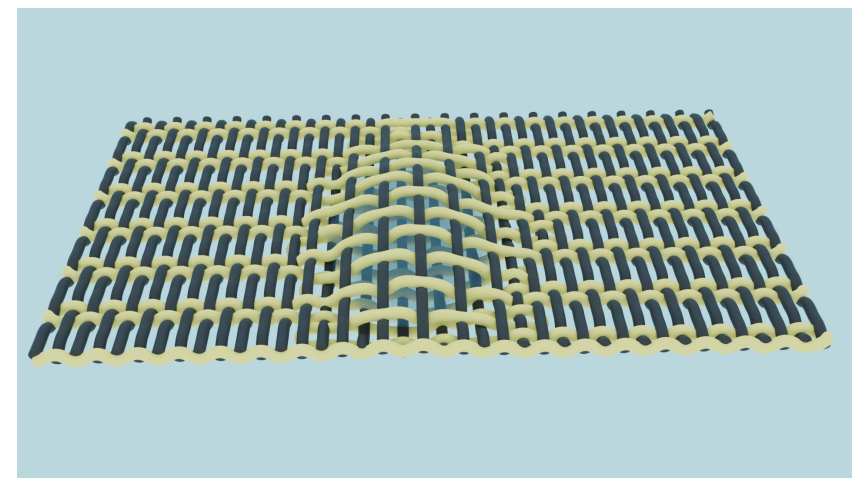

Figure 6: A diagram of double cloth weaving showing the "pocket" formed in one area of the cloth. When actually woven, each weft line would be compressed against the previous ones, resulting in the outcome shown in Fig. 7.

cloth pattern can be difficult even for intermediate-level weavers, especially if each fabric face itself has complicated patterning. Additionally, when woven on a frame loom, double cloth patterns require twice as many frames at the same complexity of face patterning; the frame quantity requirement rises if the doubled area is not rectangular. Thus double cloth is an ideal demonstration of computational jacquard capabilities.

We show a system in which an object to be incorporated (e.g. a PCB for an e-textiles project) is laid directly on the not-yet-woven part of the warp. The image is captured by the same camera that handles the heddle input, and it is rectified to represent the realworld size and shape of the object. The weaver can then apply weaving patterns to all three areas of the fabric: the single-layer area, and the front and back faces of the pocket. These patterns are then computationally composited for weavability to create the weaving pattern.

In addition to the elaborate structural patterning enabled by jacquard weaving, this system takes advantage of the loom's integrated calibrated camera system for capturing real-world input to the design process. In this case, this input allows the weaver to incorporate physical objects without the need for measuring, a hallmark strength of on-machine interaction systems.

\subsection{Collaborative Editing}

Computational mediation allows a creative process to have remotely-accessed components. We show a system in which the current weaving plan can be accessed in real time via a web server. The weaver and any number of remote collaborators can edit the plan using a web-based editing tool; edits are shared in real time, as in other online collaborative editing tools such as Google Docs.

The editing interface includes "brushes" of different weaving patterns at different sizes; any collaborator can "paint" with these and a viable weaving pattern will result. The weaver can choose which brushes to make available to collaborators; for example, they could focus on just a series of tonally gradated satin patterns. During the weaving process, the weaver can manipulate the heddles directly to alter the pattern. The weaver can choose to capture these

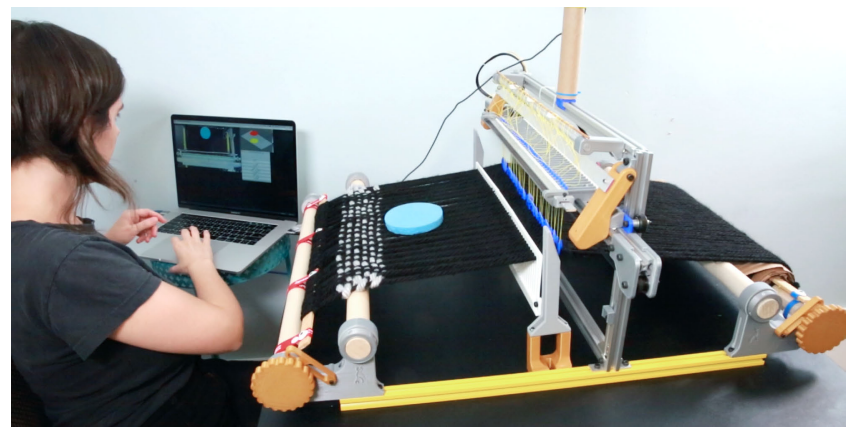

(a) The weaver places an item on the loom. The system captures and rectifies the outline of the item. The weaver allocates different weaving patterns to the two faces.

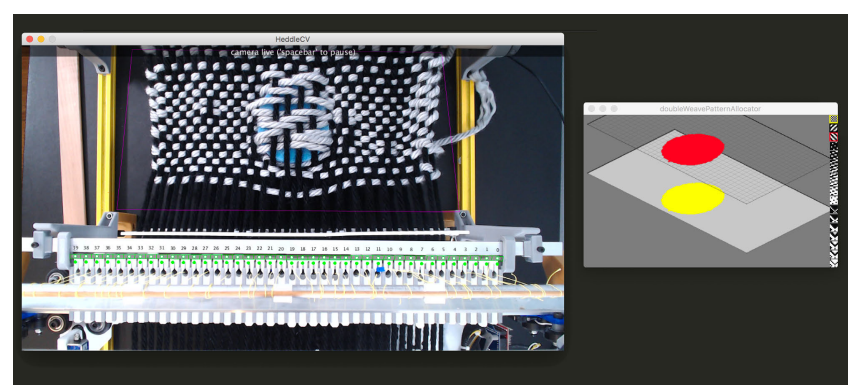

(b) A screenshot showing the camera's view of the weaving as well as the pattern allocator. The weaver has assigned a twill pattern to the upper layer and a plain weave to the lower.

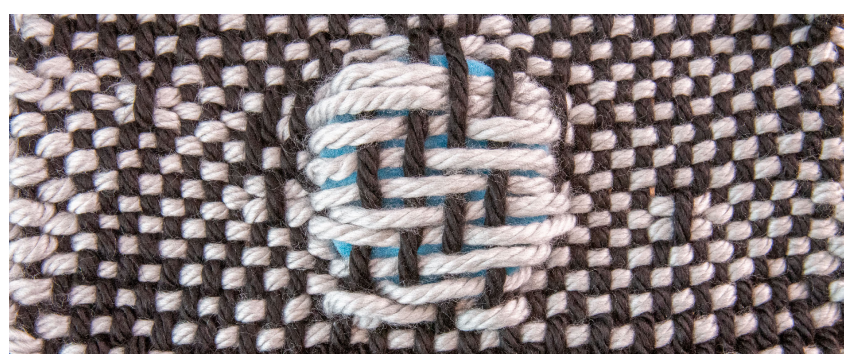

(c) The finished double cloth weaving

Figure 7: The On-Loom Input example

changes using the heddle-tracking system and send them to the server to share with their collaborators.

This system highlights how computational mediation can enable remote collaboration, as well as how simplified pattern design tools like "brush" systems can make expert tasks accessible.

\subsection{At-home Weaving}

While our loom has many applications for use in a lab or makerspace, we consider its size and cost particularly appealing for home and daily use.

As an example of the potential of integrating computational weaving into a personal weaving practice, we show a system which encodes a daily weather report into the weaving pattern. The day's temperature relative to the previous day's is shown as the shading of the pattern - more warp-dominant patterns indicate cooler, 


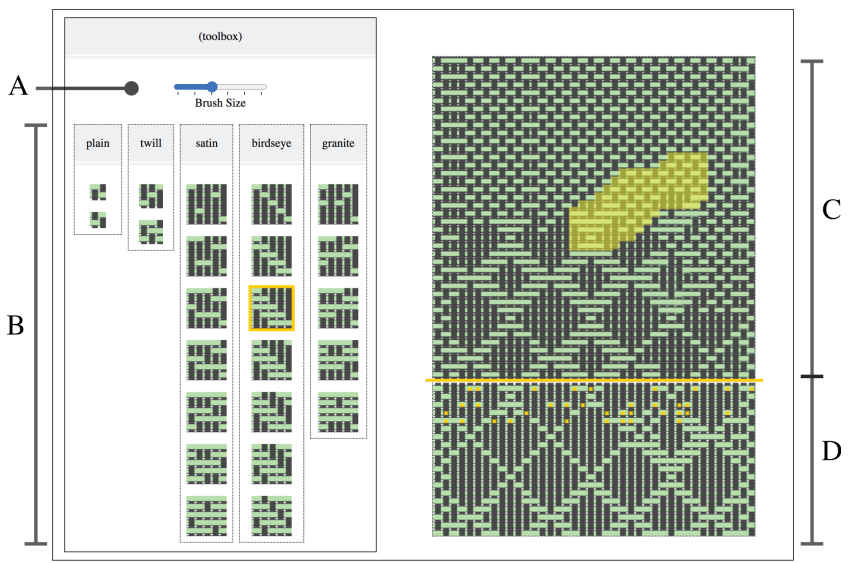

(a) The editor is a browser-based application which allows multiple users to simultaneously edit. A: "brush size" allows users to edit larger or smaller areas of the fabric. B "brushes" are loaded from a pattern repository. C: the editable weaving area. A collaborator's edit is shown highlighted in yellow. D: Below the yellow line, the rows have been physically woven and can no longer be edited. Highlighted interlacements show where the weaver manually overrode the pattern.

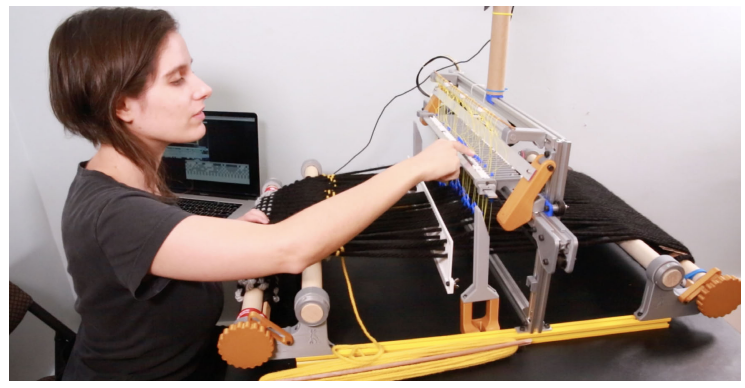

(b) The weaver overrides an area of weaving by manually changing some heddle positions.

Figure 8: Collaborative editing.

whereas more weft-dominant patterns indicate warmer - and specific weather features are indicated with different styles of pattern, such as slanting twills for rain, dappled satins for clouds, and wavy broken twills for wind.

Of course, the weaver retains control over weft yarn color choices, as well as how many rows to weave, so the resulting fabric incorporates both formal weather data as well as the weaver's own expectations and desire to spend time weaving on that day. This system demonstrates a way that the flexibility of computational jacquard weaving can extend beyond weaver-directed changes, to incorporate sources which may surprise even the weaver, or spur their creative practice.

\section{LIMITATIONS AND FUTURE WORK}

As a jacquard loom, our design supports any sequence of warp and weft interlacements. However, some fabrics require other loom features. For example, our fixed heddle spacing presents a slight limitation in being decreasingly appropriate for higher numbers of woven layers (triple cloth, quadruple cloth, etc); however, these are
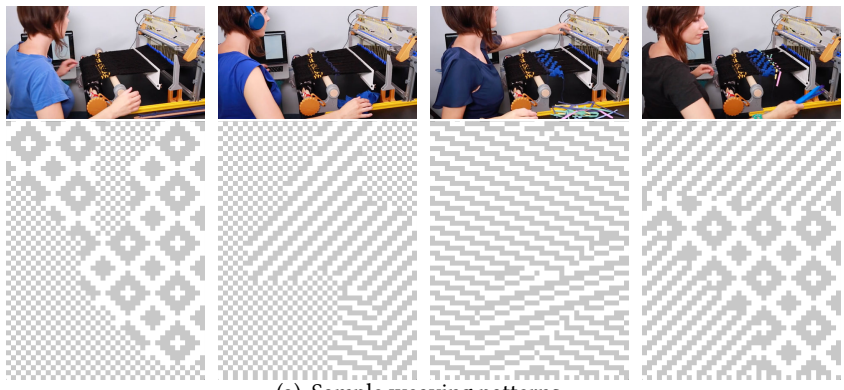

(a) Sample weaving patterns

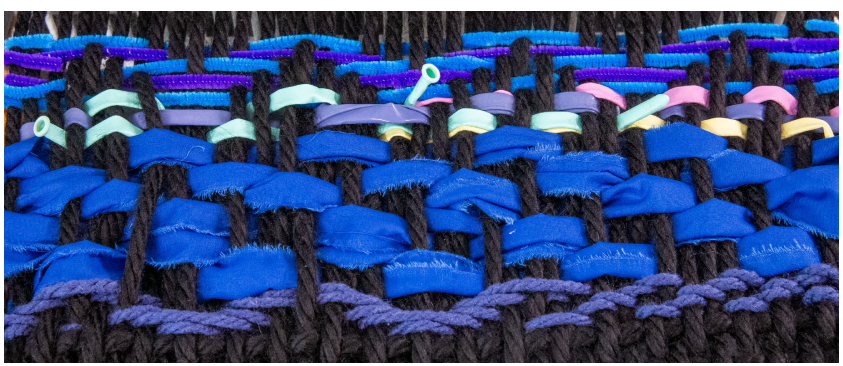

(b) An accumulated weaving.

Figure 9: Weaving can incorporate many materials: pipe cleaners, balloons, and strips of fabric are shown here as improvisational responses to generated weaving patterns seeded by daily weather conditions.

typically woven on looms with other purpose-built features such as extra warp beams.

Additionally, our 10mm warp thread spacing (center to center) is relatively large compared to most other looms. This is because we prioritized low-precision construction and easy manipulability for hands-on hybrid weaving. Combined with the loom's small overall size, chosen for convenience and portability, this spacing results in only 40 warp threads. (Of course, the serial design means that adding more warps would only require more width, not any further electronic complexity.) However, many hand looms have a much smaller spacing, such as $2.5 \mathrm{~mm}$ for a "rug loom" (typically a four- or eight-set loom as described in section 1.1). Future iterations might decrease the warp spacing in several ways: miniaturization with more-precise printing or other manufacturing approaches; additional heddle arrays above or behind, with constant mechanical complexity per frame; to an extent, tilting the heddle frames as in AVL's "dial a sett" system [8]. Each has tradeoffs but is potentially viable, and they might also be combined.

We showed design systems which focused on specific capabilities enabled by our loom. Due to the general interoperability of jacquard weaving patterns, it should be possible to use our loom with existing, broader weaving design tools as well. For example, integrating with AdaCAD [19] would enable a wide range of e-textiles design functionality.

We also showed an application incorporating double cloth weaving. These pockets are flat and are suitable for integrated components like circuit boards, conduit, or the padding shown in 
the demonstration. Multi-layered cloth weaving is an intriguing design space which is constrained by global warp tensioning (in our loom, as in most others). Individual warp tension is not typical on any loom (though one related patent exists [13]) due to the electromechanical complexity involved. Our approach to serial warp selection may also point the way toward more flexible warp tensioning, unlocking some of the 3D shaping potential that is currently dominated by knitting [31].

As an inexpensive and portable piece of fabrication equipment, our jacquard loom particularly lends itself to future exploration on the social possibilities of networked fabrication. The social role of hobby crafting is well-documented [4, 12, 40], but most computationally-enabled craft socialization is purely virtual $[10,25]$, relying on in-person meetups for the actual co-creation of objects. Especially as national lockdowns have shown us how tenuous in-person meetups can be, how might we use multiply-located personal computational fabrication as a social bridge?

\section{CONCLUSION}

We have described an inexpensive personal weaving system which uses serial selection of a passive bistable heddle mechanism to avoid the electronic complexity of industrial jacquard looms which actuate all heddles in parallel. This allows us to dramatically reduce both acquisition and maintenance costs, and stay within a tabletop form factor convenient for personal use. Our loom is explicitly designed to support and extend the activities of handweaving, which differ from those of industrial production of cloth. For example, our loom supports introduction of a broad range of possibly non-standard materials, or non-standard manipulations of the warp or weft, as well as improvisational changes to patterns and on-the-fly decisions. By also bringing the richness of computational patterning to handweaving, such as the ability to work with programmaticallydesigned double cloth structures, to record and potentially embellish improvisations, or incorporate information from remote collaborators or data feeds, we also extend the potential expressive reach of handweaving.

Our loom makes jacquard weaving accessible outside of industry and specialized textiles scholarship, with implications for e-textiles prototyping, fiber arts education, and personal craft.

\section{ACKNOWLEDGMENTS}

The authors would like to thank Sarika Bajaj and Kaushik Apte for their work on earlier prototypes, as well as Kurt Schaefer for discussing his loom project with us. David Renshaw is an invaluable photographer. This research was supported in part by National Science Foundation Grant IIS-1718651.

\section{REFERENCES}

[1] Lea Albaugh. 2018. Little Looms. http://lea.zone/little-looms.html. (accessed 2020-12-28).

[2] Lea Albaugh, April Grow, Chenxi Liu, James McCann, Gillian Smith, and Jennifer Mankoff. 2016. Threadsteading: Playful Interaction for Textile Fabrication Devices. In Proceedings of the 2016 CHI Conference Extended Abstracts on Human Factors in Computing Systems - CHI EA '16. ACM Press, San Jose, California, USA, 285-288. https://doi.org/10.1145/2851581.2889466

[3] Lea Albaugh, Scott E. Hudson, Lining Yao, and Laura Devendorf. 2020. Investigating Underdetermination Through Interactive Computational Handweaving. In Proceedings of the 2020 ACM Designing Interactive Systems Conference (DIS
'20). Association for Computing Machinery, New York, NY, USA, 1033-1046. https://doi.org/10.1145/3357236.3395538
[4] Ash Amin and Joanne Roberts. 2008. Knowing in Action: Beyond Communities of Practice. Research Policy 37, 2 (March 2008), 353-369. https://doi.org/10.1016/ j.respol.2007.11.003

[5] Kristina Andersen, Ron Wakkary, Laura Devendorf, and Alex McLean. 2019. Digital Crafts-Machine-Ship: Creative Collaborations with Machines. Interactions 27, 1 (Dec. 2019), 30-35. https://doi.org/10.1145/3373644

[6] AVL. 2013. Little Weaver Manual. https://www.avlusa.com/downloads/LW Manual_Current.pdf. (accessed 2020-12-29).

[7] AVL. 2017. Price List September 2017. http://www.avlusa.com/downloads/ AVLPriceList.pdf. (accessed 2017-10-10).

[8] AVL Looms Inc. 2020. Jacq3G Jacquard Loom. https://avllooms.com/products/ jacq3g. (accessed 2020-09-03).

[9] Margarita Benitez, Nathan Clark, Chris Yanc, Markus Vogl, Ed Bennett, and Gary Shimomura. 2013. Open Source Jacquard Loom Project. http://www.osloom.org/. (accessed 2020-12-29).

[10] Leonardo Bonanni and Amanda Parkes. 2010. Virtual Guilds: Collective Intelligence and the Future of Craft. The fournal of Modern Craft 3, 2 (July 2010), 179-190. https://doi.org/10.2752/174967810X12774789403564

[11] Toni Buckby. 2018. DIY Laser Cut Rigid Heddle Loom. https://www.instructables. com/DIY-Laser-Cut-Rigid-Heddle-Loom-Part-1-Building-th/. (accessed 202012-28).

[12] Leah Buechley, Daniela K. Rosner, Eric Paulos, and Amanda Williams. 2009. DIY for CHI: Methods, Communities, and Values of Reuse and Customization. In Proceedings of the 27th International Conference Extended Abstracts on Human Factors in Computing Systems - CHI EA '09. ACM Press, Boston, MA, USA, 4823. https://doi.org/10.1145/1520340.1520750

[13] Alexander Busgen. 1999. Woven Fabric Having a Bulging Zone and Method and Apparatus of Forming Same. Patent No. 6,000,442, Filed Mar. 29, 1996, Issued Dec. 14, 1999.

[14] Laura Devendorf. 2016. Strange\& Unstable Fabrication. Ph.D. Dissertation. University of California, Berkeley, Berkeley, CA.

[15] Digital Weaving Norway. 2020. TC2 Loom | Digital Weaving Machine. https: //www.digitalweaving.no/tc2-loom/. (accessed 2020-09-03).

[16] James Essinger. 2004. Jacquard's Web: How a Hand-Loom Led to the Birth of the Information Age. Oxford University Press, Oxford, UK.

[17] Marianne Fairbanks, Erica Hess, Kat Bunke, and Sofia Hagström Møller. 2020. HELLO! LOOMS. https://www.weavinglab.com/tools. (accessed 2020-12-29).

[18] Sean Follmer, David Carr, Emily Lovell, and Hiroshi Ishii. 2010. CopyCAD: Remixing Physical Objects with Copy and Paste from the Real World. In Adjunct Proceedings of the 23nd Annual ACM Symposium on User Interface Software and Technology (UIST '10). Association for Computing Machinery, New York, NY, USA, 381-382. https://doi.org/10.1145/1866218.1866230

[19] Mikhaila Friske, Shanel Wu, and Laura Devendorf. 2019. AdaCAD: Crafting Software For Smart Textiles Design. In Proceedings of the 2019 CHI Conference on Human Factors in Computing Systems - CHI '19. ACM Press, Glasgow, Scotland Uk, 1-13. https://doi.org/10.1145/3290605.3300575

[20] Nur Al-huda Hamdan, Simon Voelker, and Jan Borchers. 2018. Sketch\&Stitch: Interactive Embroidery for E-Textiles. In Proceedings of the 2018 CHI Conference on Human Factors in Computing Systems - CHI '18. ACM Press, Montreal QC, Canada, 1-13. https://doi.org/10.1145/3173574.3173656

[21] BL Hardy, M-H Moncel, Céline Kerfant, Matthieu Lebon, Ludovic Bellot-Gurlet, and Nicolas Mélard. 2020. Direct Evidence of Neanderthal Fibre Technology and Its Cognitive and Behavioral Implications. Scientific reports 10, 1 (2020), 1-9.

[22] Claire Harvey, Emily Holtzman, Joy Ko, Brooks Hagan, Rundong Wu, Steve Marschner, and David Kessler. 2019. Weaving Objects: Spatial Design and Functionality of 3D-Woven Textiles. Leonardo 52, 4 (Aug. 2019), 381-388. https://doi.org/10.1162/leon_a_01780

[23] Hot World Media GmbH. 2020. Repetier-Firmware. https://www.repetier.com/ documentation/repetier-firmware/. (accessed 2020-09-03).

[24] Hot World Media GmbH. 2020. Repetier-Firmware Configuration Tool v0.92.9. https://www.repetier.com/firmware/v092/. (accessed 2020-09-03)

[25] Sal Humphreys. 2009. The Economies within an Online Social Network Market: A Case Study of Ravelry. In Communication, Creativity and Global Citizenship. Australian and New Zealand Communication Association, Brisbane, Australia, $8-10$.

[26] Johnny Russell. 2020. RAMPS 1.4. https://reprap.org/wiki/RAMPS_1.4. (accessed 2020-09-03).

[27] Marinos Koutsomichalis and Afroditi Psarra. 2015. Computer-Aided Weaving: From Numerical Data to Generative Textiles. In Electronic Visualisation and the Arts (EVA 2015). BCS Learning \& Development Ltd., London, UK, 122-123. https://doi.org/10.14236/ewic/eva2015.59

[28] Pamela Liou. 2015. Doti the Desktop Loom. https://pamelaliou.com/doti.html. (accessed 2020-12-29)

[29] Shiran Magrisso, Moran Mizrahi, and Amit Zoran. 2018. Digital Joinery For Hybrid Carpentry. In Proceedings of the 2018 CHI Conference on Human Factors in Computing Systems (CHI '18). Association for Computing Machinery, New York, NY, USA, 1-11. https://doi.org/10.1145/3173574.3173741 
[30] David NG McCallum. 2018. Glitching the Fabric: Strategies of New Media Art Applied to the Codes of Knitting and Weaving. Ph.D. Dissertation. Unpublished.

[31] James McCann, Lea Albaugh, Vidya Narayanan, April Grow, Wojciech Matusik, Jennifer Mankoff, and Jessica Hodgins. 2016. A Compiler for 3D Machine Knitting. ACM Transactions on Graphics 35, 4 (July 2016), 49:1-49:11. https://doi.org/10. $1145 / 2897824.2925940$

[32] Jennifer Moore. 2010. The Weaver's Studio: Doubleweave. Interweave, USA.

[33] Ilan Moyer. 2013. 24-Bit Friendship Loom. http://web.mit.edu/imoyer/www/ portfolio/jacquard/index.html. (accessed 2020-12-29)

[34] Stefanie Mueller. 2016. Interacting with Personal Fabrication Devices. PhD Thesis Universität Potsdam.

[35] Jerry Nicholls. 2018. Weav3r LEGO Loom Weaving a Scanned Pattern, Unloaded. https://www.youtube.com/watch?v=7pjrM-yqhVA. (accessed 2020-09-03).

[36] Paul R O'Connor. 2006. Double Weave - A Retrospective. In Complex Weavers Seminars. Hope College, Holland, Michigan, 34

[37] Daniëlle Ooms, Nick Voskuil, Kristina Andersen, and Hanna Ottilia Wallner 2020. Ruta, a Loom for Making Sense of Industrial Weaving. In Companion Publication of the 2020 ACM Designing Interactive Systems Conference (DIS' 20 Companion). Association for Computing Machinery, Eindhoven, Netherlands, 337-340. https://doi.org/10.1145/3393914.3395815

[38] Neri Oxman. 2007. Digital Craft: Fabrication Based Design in the Age of Digital Production. In Workshop Proceedings for Ubicomp 2007: International Conference on Ubiquitous Computing. ACM, Innsbruck, Austria, 534-538.

[39] Emanuel Anthony Posselt. 1887. The Jacquard Machine Analyzed and Explained: With an Appendix on the Preparation of Jacquard Cards. Pennsylvania museum and school of industrial art, Philadelphia, PA.

[40] Elena Prigoda and Pamela J. McKenzie. 2007. Purls of Wisdom: A Collectivist Study of Human Information Behaviour in a Public Library Knitting Group Journal of Documentation 63, 1 (Jan. 2007), 90-114. https://doi.org/10.1108/ 00220410710723902

[41] Brian Randell. 1994. The Origins of Computer Programming. IEEE Annals of the History of Computing 16, 4 (1994), 6-14.

[42] Daniela K. Rosner, Samantha Shorey, Brock R. Craft, and Helen Remick. 2018. Making Core Memory: Design Inquiry into Gendered Legacies of Engineering and Craftwork. In Proceedings of the 2018 CHI Conference on Human Factors in Computing Systems. ACM, Montreal QC Canada, 1-13. https://doi.org/10.1145/ 3173574.3174105

[43] Kurt Schaefer. 2019. Making a DIY Computer Controlled Loom. https:// retrotechjournal.com/2019/06/17/making-a-diy-computer-controlled-loom/. (accessed 2020-09-03).
[44] Alice Schlein. 1994. Network Drafting: An Introduction. Bridgewater Press, Greenville, SC, USA.

[45] Alice Schlein and Bhakti Ziek. 2006. The Woven Pixel: Designing for facquard and Dobby Looms Using Photoshop®. Bridgewater Press, Greenville, SC, USA.

[46] Olga Soffer. 2004. Recovering Perishable Technologies through Use Wear on Tools: Preliminary Evidence for Upper Paleolithic Weaving and Net Making. Current Anthropology 45, 3 (2004), 407-413.

[47] Anne Sullivan, Joshua Allen McCoy, Sarah Hendricks, and Brittany Williams. 2018. Loominary: Crafting Tangible Artifacts from Player Narrative. In Proceedings of the Twelfth International Conference on Tangible, Embedded, and Embodied Interaction. ACM, Stockholm Sweden, 443-450. https://doi.org/10.1145/3173225. 3173249

[48] Rundong Tian, Sarah Sterman, Ethan Chiou, Jeremy Warner, and Eric Paulos. 2018. MatchSticks: Woodworking through Improvisational Digital Fabrication. In Proceedings of the 2018 CHI Conference on Human Factors in Computing Systems (CHI '18). Association for Computing Machinery, Montreal QC, Canada, 1-12. https://doi.org/10.1145/3173574.3173723

[49] Cesar Armando Torres. 2019. Hybrid Aesthetics: Bridging Material Practices and Digital Fabrication through Computational Crafting Proxies. Ph.D. Dissertation. UC Berkeley.

[50] Van De Wiele. 2020. VSi32. https://www.vandewiele.be/en/activities/vsi32. (accessed 2020-12-29).

[51] M. B. Windeknecht and T. G. Windeknecht. 1980. Microcomputer Graphics and the Color-and-Weave Effect in Handweaving. In Proceedings of the 18th Annual Southeast Regional Conference (ACM-SE 18). Association for Computing Machinery, Tallahassee, Florida, 174-179. https://doi.org/10.1145/503838.503866

[52] Matthijs Witsenburg. 2016. Laser Cut Plywood Bandweaving Loom. https://www.hackster.io/matthijs-witsenburg/laser-cut-plywoodbandweaving-loom-32e22c. (accessed 2020-12-28).

[53] Mayu M. Yamashita, Junichi Yamaoka, and Yasuaki Kakehi. 2013. Enchanted Scissors: A Scissor Interface for Support in Cutting and Interactive Fabrication. In ACM SIGGRAPH 2013 Posters on - SIGGRAPH '13. ACM Press, Anaheim, California, 1. https://doi.org/10.1145/2503385.2503422

[54] Amit Zoran and Joseph A. Paradiso. 2013. FreeD: A Freehand Digital Sculpting Tool. In Proceedings of the SIGCHI Conference on Human Factors in Computing Systems - CHI '13. ACM Press, Paris, France, 2613. https://doi.org/10.1145/ 2470654.2481361 